\title{
Motivação para utilização da Extreme Programming
}

\author{
Eduardo Júnio de Oliveira; Cayley Guimarães \\ eduardojuniol@gmail.com; profcayley@yahoo.com.br \\ Centro Universitário de Belo Horizonte (UNI-BH). \\ 30.455-610 - Belo Horizonte - MG - Brasil
}

\begin{abstract}
Resumo: Este artigo tem por objetivo apresentar a metodologia de melhoria para desenvolvimento de software denominada Extreme Programming. Serão abordadas, de forma resumida, as práticas, valores, e os papéis disponiveis a cada integrante de uma equipe de $X P$. Alguns comparativos serão feitas com outras metodologias ao decorrer do trabalho visando as propriedades que definem a Extreme Programming.
\end{abstract}

Palavras-chave: Extreme Programming , XP, Engenharia de Software, Qualidade de Software, Metodologia de Desenvolvimento, Valores de Desenvolvimento, Práticas de Desenvolvimento.

Abstract: This article aims to present the methodology of improvement for software development called Extreme Programming. Will be addressed in summary form, practices, values, and roles available to each member of a team of XP. Some comparisons are made with other methods to place of work to the properties that define the Extreme Programming.

\section{INTRODUÇ̃̃o}

Com a evolução do setor de informática, a indústria de software vem passando por grandes transformações, inovações e novos desafios. Entre estes desafios, o desenvolvimento de softwares com qualidade, no menor tempo possível e que atendam as necessidades dos clientes são importantes [Telles 2004].

Com estes novos desafios a indústria de software passou a dar valor a algumas áreas da informática, como a engenharia de software e qualidade de software, com intuito de atender as exigências do mercado e consequentemente as necessidades dos clientes.

As empresas do setor de informática começaram a utilizar metodologias de desenvolvimento de software, adotou métricas e padrões para alcançar níveis aceitáveis de qualidade, prever custos e prazos em seus projetos. Porém ainda são poucos os projetos que conseguem obter pleno sucesso em seu desenvolvimento, onde prazo e orçamento estabelecidos e as necessidades do cliente sejam realmente atendidas. [Beck 2004]

Pesquisa feitas pelo Standish Group International em 1994, um pouco antes da adoção de metodologia de desenvolvimento pelas indústrias, apontam que apenas 16, $2 \%$ dos projetos de software atingiam sucesso (prazo, orçamento e funcionalidades atendidas). Em 2002 esta taxa havia subido para $34 \%$, ou seja, um aumento de $100 \%$ em 8 anos. Mas estas taxas ainda se encontram muito aquém do esperado pelo mercado.

As metodologias utilizadas nos projetos pesquisados eram as mais variadas, podemos citar modelo em cascata, modelo iterativo e alguns com modelo em prototipação. Será relatado neste documento e utilizado o termo desenvolvimento tradicional para os projetos que se utilizam do modelo em cascata e todos os outros que se baseiam nele.

Uma destas metodologias de desenvolvimento ágil é o Extreme Programming, metodologia que visa a qualidade do software a ser desenvolvido, que atenda as reais necessidades do cliente e seja entregue dentro do prazo definido. Esta metodologia é assunto a ser descrito a seguir.

\section{Extreme Programming (XP)}

Extreme Programming ou XP, como também é conhecida, é uma metodologia voltada para projetos cujos requisitos mudem com 
freqüência, utilizem desenvolvimento orientado a objetos, equipes de até 12 desenvolvedores e desenvolvimento incremental. Tem como objetivo ter agilidade, com qualidade e que atenda as necessidades do cliente nas fases de desenvolvimento de software. Alguns praticantes definem a XP como a prática e a perseguição da mais clara simplicidade, aplicado ao desenvolvimento de software. [Telles 2004]

A XP Busca o máximo de valor a cada dia de trabalho da equipe para o seu cliente. Em um curto espaço de tempo o cliente terá um produto que possa ser utilizado, podendo aprender com o mesmo e reavaliar se o que foi desenvolvido é realmente o desejado para aquele cenário, necessidade. Por ser uma metodologia recente, a XP sofre mudanças em suas concepções e, portanto, é comum encontrar variações. A adaptação ao ambiente de desenvolvimento deve ser levada em conta, se um valor trouxer mais prejuízos do que benefícios é necessário reavaliar a utilização desta metodologia. [Telles 2004]

A XP é organizada em torno de um conjunto de práticas e valores que atuam perfeitamente para assegurar um alto retorno do investimento efetuado pelo cliente. Os valores (Feedback, Coragem, simplicidade e Comunicação) são as diretrizes da XP. Eles definirão as atitudes das equipes e as principais prioridades da metodologia Já as práticas da XP(cliente sempre presente, Jogo de planejamento, Stand up meeting, programação em par, refactoring que é o processo de reescrever um programa de computador para melhorar sua estrutura ou legibilidade, preservando seu comportamento, código coletivo, padrões de desenvolvimento, desenvolvimento guiado por testes, design simples, metáforas, releases curtos, ritmo sustentável e integração continua)é um conjunto de atividades que deverão ser seguidas pelas equipes que desejam utilizar esta metodologia. [Beck 2004].

\section{Motivação para utilização}

A metodologia XP atende as necessidades do setor de informática nos quesitos qualidade de software, satisfação do cliente e ganhos produtivos na empresa no processo de fabricação de um software.

Neste estudo, será adotada uma ferramenta para avaliação de alguns estudos de caso de adoção dos valores e práticas desta metodologia, ou seja será descrito estudo sucinto de alguns projetos de empresas desenvolvedoras de software que utilizam esta metodologia como ferramenta para elaboração, analise, validação e desenvolvimento de um software. A ferramenta que será abordada para avaliação do uso da XP por projetos desenvolvidos e em andamento é uma adaptação do Framework que é elaborado e explorado por Lucas Layman (2004) que será descrito a seguir.

\section{Metodologia}

Para os estudos de casos apresentados neste artigo examinaremos o bom e desejado resultado de negócios resultante da adoção da metodologia XP. Tais alterações, como ganho na produtividade, aumento da qualidade do software a ser desenvolvido e satisfação do cliente são aspectos a ser considerados e avaliados pelo uso desta ferramenta.

Múltiplos estudos de caso serão necessários para fornecer resultados suficientes e avaliativos para que possamos tirar qualquer conclusões sobre a eficácia da XP. De acordo com as orientações do Goal Question Metric paradigma, o objetivo é construir teorias sobre se o negócios relacionados com os resultados de uma equipe que utilizam praticas e valores da XP podem ou não obter melhoria para $o$ desenvolvimento do produto a ser construído para o cliente. Esta meta foi refinada em perguntas sobre: 
1. Com a utilização da XP no Prélançamento do produto como esta avaliação da qualidade do mesmo?

2. Após a entrega com esta a avaliação de qualidade do produto desenvolvido utilizando a XP?

3. Com a adoção da XP como pode ser avaliada a produtividade da equipe de programadores?

4. Utilizando esta ferramenta como esta a satisfação do cliente?

5. Avaliação da moral da equipe com a adoção da ferramenta para o desenvolvimento do projeto.

Para orientar e facilitar a nossa investigação, nosso estudo de caso de projetos desenvolvido com aplicação desta metodologia será adotado um modelo de Framework apresentado por Luca Layman (2004) trata-se um framework sobre a aplicação e uso da Extreme Programming no desenvolvimento de um software, como é demonstrado na figura 1 .

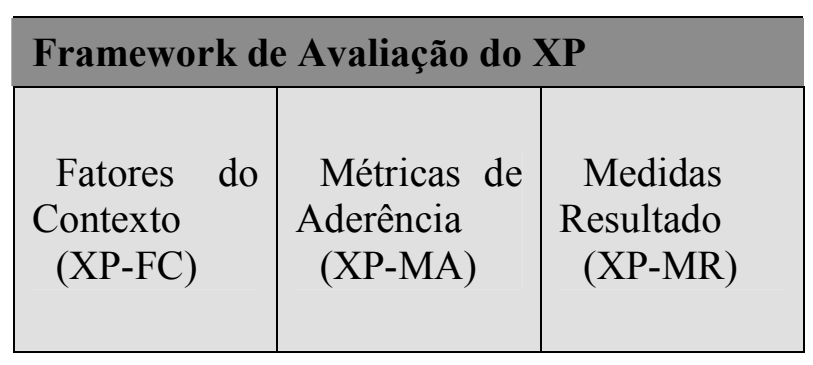

Figura1 - Framework de Avaliação do XP. Adaptado de [3];

Este Framework, visto na Figura 1, a ser adotado nesta pesquisa, possui 3 etapas: na primeira parte, Fatores de Contexto (XP-FC), são recolhidas informações essenciais sobre $\mathrm{O}$ projeto a ser desenvolvido o contexto, tamanho do projeto, tamanho da equipe envolvida, na segunda etapa conhecida com Métricas de Aderência (XP-MA) quando é utilizada estas métricas objetivas e subjetivas para expressar concretamente e comparativamente a medida em que uma equipe utiliza as métricas e valores da XP. Ao examinar vários casos de usos que utilizam a XP, o XP-MA também permitir aos investigadores de desenvolver as interações e dependências entre o XP práticas e em que medida as práticas podem ser separadas ou eliminadas. [Astels et al 2002]

Na terceira e última parte do Framework apresentado, o Medidas Resultado (XP-MR), permite avaliar a atividade relacionada com os resultados (produtividade, qualidade, etc) através de um conjunto completo ou parcial das práticas adotada da XP. Além disso, realizar entrevistas com membros da equipe e clientes para ajudar a compreender a equipe sobre a adoção do XP e a satisfação do cliente com o projeto que esta sendo modelado para atender a sua necessidade. [Khun 2009]

\section{Estudos dos Casos de Uso}

Temos concluída a análise de dois estudos de casos estruturados usando o Framework de Avaliação do XP. Para os resultados listados abaixo, com base no estudo desenvolvido por Lucas Layman, medições são normalizadas com os antigos lançamentos para proteger informação privada. Tanto a satisfação do cliente e moral é avaliada utilizando meios subjetivos (entrevista e cliente programador inquérito, respectivamente). Estas medidas podem ser objeto para além dos fatores que influenciam as práticas XP, embora o entrevistas e perguntas do inquérito foram estruturadas de forma a reduzir o impacto destes fatores, tanto quanto possível. Nosso pós-release qualidade os resultados são limitados pela falta de uma medida de cliente uso do produto durante o defeito coleção períodos.

Primeiro caso de uso a ser avaliado na IBM, empresa que presta soluções no setor de informática. Foi realizado estudo do caso de uso com uma pequena equipe de 7 a 11 membros, o produto desenvolvido era o Servlet / XML. 
Medidas de Resultados do caso de uso estudo pela IBM é apresentado na tabela 1 seguinte.

\begin{tabular}{|l|l|l|}
\hline $\begin{array}{l}\text { Medidas de } \\
\text { Resultados da XP }\end{array}$ & $\begin{array}{l}\text { Sem uso } \\
\text { XP }\end{array}$ & $\begin{array}{l}\text { Após } \\
\text { uso XP }\end{array}$ \\
\hline $\begin{array}{l}\text { Qualidade Pré-testes } \\
\text { (Testes edefeitos por } \\
\text { linha de código } \\
\text { programado). }\end{array}$ & 1.0 & 0.50 \\
\hline $\begin{array}{l}\text { Qualidade Pós-entrega } \\
\text { (Uso de 6 meses) }\end{array}$ & 1.0 & 0.61 \\
\hline Produtividade & & 1.34 \\
\hline Colaborador & 1.0 & 1.7 \\
\hline Colaborador/mês & 1.0 & 1.92 \\
\hline $\begin{array}{l}\text { Parâmetros de linha } \\
\text { de código programada. }\end{array}$ & 1.0 & Alta \\
\hline Satisfação do Cliente & $\begin{array}{l}\text { Sem } \\
\text { Avaliação }\end{array}$ & 1.11 \\
\hline Moral da equipe & 1.0 & \\
\hline
\end{tabular}

Tabela 1 - Medidas Resultados IBM

Outro caso de estudo apresentado por Luca Layman é sobre a Sabre Airline Solutions equipe se limitava de 6 a 10 pessoas, é desenvolvido um software empresarial no ambiente GUI . A seguir a tabela 2 com base nos resultados obtidos.

\begin{tabular}{|l|l|l|}
\hline $\begin{array}{l}\text { Medidas de } \\
\text { Resultados da XP }\end{array}$ & $\begin{array}{l}\text { Sem uso } \\
\text { XP }\end{array}$ & $\begin{array}{l}\text { Após } \\
\text { uso XP }\end{array}$ \\
\hline $\begin{array}{l}\text { Qualidade Pré-testes } \\
\text { (Testes edefeitos por } \\
\text { linha de codigo } \\
\text { programado). }\end{array}$ & 1.0 & 0.35 \\
\hline $\begin{array}{l}\text { Qualidade Pos-entrega } \\
\text { (Uso de 6 meses) }\end{array}$ & 1.0 & 0.64 \\
\hline Produtividade & 1.0 & 1.46 \\
\hline Colaborador/mês & 1.0 & 1.92 \\
\hline $\begin{array}{l}\text { Parâmetros de linha } \\
\text { de codigo programada. }\end{array}$ & $\begin{array}{l}\text { Sem } \\
\text { Avaliação }\end{array}$ & Alta \\
\hline Satisfação do Cliente & $\begin{array}{l}\text { Sem } \\
\text { Avaliação }\end{array}$ & $68.1 \%$ \\
\hline Moral da equipe
\end{tabular}

Tabela 2 - Medidas Resultados Airline
Outro estudo de caso de que foi avaliado em cima das cinco perguntas elaboradas é a Studio Sol, que é uma empresa mineira com 10 anos de atuação em entretenimento para a web. Acreditando sempre na inovação, praticidade e no foco para o usuário, desenvolveu vários sites que são sucessos principalmente entre o público jovem. A empresa que desenvolve produtos voltados para ambiente WEB e possui alguns sites com grande acesso na atualidade como o cifrasclub.com.br. A equipe de desenvolvimento que atualmente colabora para a criação de aplicativos gira em torno de 4 a 9 membros.

Na Studio Sol, é utilizado o XP para o desenvolvimento de web sites. O produto já se encontra no servidor onde ele será hospedado e nesse processo os testes são feitos. Por não trabalhar com clientes diretos, os testes são realizados pela equipe mesmo e, caso esteja tudo correto, ele vai pro ar. Após o lançamento, é levantado um acompanhamento constante para verificar como está o número de acessos, se o servidor está respondendo bem, o que pode ser melhorado em termos de desempenho, como está a saúde do banco de dados, entre outros tantos pontos que são avaliados.

A qualidade do produto tem melhorado bastante. Com os testes e os conhecimentos adquiridos pode-se perceber um crescimento considerável no processo de desenvolvimento e o resultado final a cada dia é mais satisfatório.

Como já descrito, depois de lançado o produto, fazemos um monitoramento constante da performance do servidor, do banco de dados e do tempo de carregamento do site. Podemos observar que muitos problemas que tínhamos anteriormente, como "queda" do servidor por causa do grande número de acessos, já não ocorrem mais. Uma avaliação melhor é feita durante o desenvolvimento, técnicas para armazenar páginas ou dados em cache são utilizadas e, com isso, a qualidade e o tempo de 
resposta dos nossos produtos tem melhorado bastante com o tempo.

O processo de implantação do XP ainda esta sendo realizado na empresa. Ainda não tenho como ser avaliado a melhora da produtividade, pois agora que esta sendo habituado a essa forma de trabalho e, portanto, acredita-se que será a partir de então que teremos uma noção produtiva está à equipe.

O sistema de trabalho adotado na Studio Sol é um pouco diferente das outras empresas. Não trabalha diretamente com cliente externo e ganhamos com publicidade nos nossos produtos e parcerias com terceiros. O que é produzido surge de alguma idéia interna na qual é trabalhada em cima e criado um novo produto ou uma nova versão para algum já existente. Por isso, não tenho como falar da satisfação do cliente. Mas posso-se concluir que, como somos nossos próprios clientes, a cada novo lançamento ficamos mais satisfeitos com o que produzimos, pois vemos um aumento na complexidade no trabalho desenvolvido, mas estamos conseguindo manter a qualidade de antes.

A equipe tem respondido bem ao novo método de trabalho e tem se mostrado mais animada com o passar do tempo, pois com o XP é possível obter um resultado mais rapidamente e a coisa que um programador mais gosta de ver é o que ele fez funcionando certinho ou, na gíria de programador, "funcionando redondo". Isso tem deixado a equipe motivada e disposta a desenvolver mais o trabalho.

Outra empresa entrevista pela utilização da XP é a Takenet, empresa pioneira no mercado de SVA (Serviços de Valor Agregado) no Brasil, solidifica sua liderança como provedora de soluções móveis. Fundada em 1999, a empresa sediada em Belo Horizonte, é referência no mercado de SVA Latino Americano. Está integrada a todas as operadoras
Brasileiras e Chilenas e possui o maior portfólio de produtos e serviços atendendo a todos os participantes desse mercado, de provedores de conteúdo a veículos de mídia.

A empresa conta com a colaboração de mais de 85 profissionais nos escritórios de Belo Horizonte, São Paulo e Santiago do Chile, dos quais cerca de 50 trabalham na área de Pesquisa e Desenvolvimento.

Como principal enabler de tecnologia de SVA da América Latina, a Takenet presta vários serviços como: integração, distribuição de conteúdo, criação e gestão de portais móveis e soluções para Mobile Marketing. A Takenet também desenvolve e licencia diversas plataformas que garantem a qualidade, o desempenho e a segurança da conexão entre usuários de celular, operadoras e parceiros. [www.takenet.com.br]

A Takenet fez a implantação desta metodologia porém não continuou sua utilização pois não era aplicada diretamente para o tipo de relacionamento que se tem no desenvolvimento dos projetos que são solicitados, as equipes de trabalho e o cliente. $\mathrm{Na}$ Takenet a equipe de programação não possui um relacionamento acíduo e participante com o cliente pois não tem esta necessidade pelos projetos que são desenvolvidos. Sendo assim não obteve resultados concretos sobre a utilização desta metodologia para desenvolvimento de software.

De acordo com a necessidade e aplicação de uma metodologia para desenvolvimento de software a XP foi trocada na Takenet pelo processo escolhido (SCRUM) pois apresentava maior simplicidade e maior facilidade de gerenciamento, com opções de maior transparência tanto para os diretamente envolvidos no projeto quanto para personagens externos como diretores, a ausência do cliente e toda a equipe envolvida. 
O tempo de uso da XP foi relativamente extenso (durou aproximadamente três anos), porém nunca oficial, era utilizado informal sem validação e comprimento de todas as métricas $\mathrm{e}$ valores que a metodologia aplica., para ser avaliada os demais requisitos solicitados no processo de avaliação ficam impossibilitados.

Porém da nova metodologia que esta sendo aplicada no desenvolvimento de projetos na Takenet já possui 1 (um) ano de utilização do SCRUM, que é bastante transparente, simples e objetiva. Transparente sob o ponto de vista de, literalmente, expor praticamente em tempo real o progresso da equipe no projeto em questão. simples e objetiva no que diz respeito à parte burocrática e documentada da sua utilização, bastando apenas um painel onde as tarefas se encontram e algumas reuniões de acompanhamento desse projeto.

No entanto no cenário de desenvolvimento de projetos que é consolidado pela Takenet a medotologia XP é aceitável, porém não mais utilizado na empresa devido o cenário que se encontra o cliente, equipe de trabalho e a própria Takenet.

\section{CONCLUSÕES}

A metodologia Extreme Programming utilizada para de desenvolvimento de software, pode ser considerada extremamente recente no cenário de criação de software, porém vem acompanhando as necessidades dos desenvolvedores e o relacionamento dos clientes com as empresas de software pelo mundo.

Uma empresa ao utilizar este processo por completo, só estará agregando valor aos seus negócios e melhorando o ambiente de seus colaboradores e clientes, tratando-os como pessoas e parceiros. Contudo é necessário primeiramente realizar um estudo sobre a empresa e seus clientes para que haja aplicação correta da metodologia visando ter um custo benefício tanto para lado do cliente quanto para o lado da empresa.

Neste trabalho foi apresentados estudos de casos de usos da XP tendo uma aceitação em 3 projetos a favor mas tendo também uma rejeição já na implantação na empresa Takenet.

\section{AGRADECIMENTOS}

Gostaria de agradecer ao orientador deste trabalho Sr. Cayley Guimarães que a todo o momento acreditou na concretização do mesmo e pelo conhecimento adquirido junto ao desenvolvimento deste artigo.

\section{REFERÊNCIAS}

ASTELS, David; MILLER, Granville; NOVAK, Miroslav. Extreme Programming: Guia prático. Rio de Janeiro- RJ: Campus, 2002.

BECK, Kent. Programação extrema (XP) explicada: acolha as mudanças. Porto Alegre RS: Bookman, 2004.

TELES, Vinícius Manhães. UM ESTUDO DE CASO DA ADOÇÃO DAS PRÁTICAS E VALORES DO EXTREME PROGRAMMING. (Dissertação de Mestrado). Disponível em: $<$ http://www.improveit.com.br/xp/dissertacaoXP .pdf $>$ Acesso em 12 maio 2009

LAYMAN, Lucas, "Empirical Investigation of the Impact of Extreme Programming Practices on Software Projects," Companion to the 19th Annual ACM Conference on Object-Oriented Programming, Systems, Languages, and Applications (OOPSLA '04), Vancouver, Bc, October 24-28, 2004, pp. 328-329.

KUHN, G.R.. PAMPLONA, V.F, Apresentando XP.Disponívelem:http://www.javafree.org/conte nt/view.jf?idC ontent=5> Acesso em 15 julho. 2009 